\title{
A Detection of the Quality of Sound Gear based on Digital Signal Processing Techniques
}

\author{
Benedictor Alexander Nguchu \\ Tianjin University of Technology and Education, School of Electronics Engineering, \\ Address: 1310 Dagunan Road, Hexi, Tianjin, P. R. China
}

\begin{abstract}
Despite the fact that products in the industries are made by the same formula, the imperfections and dissimilarities between the produced products are influenced by noises and mechanical connections, moreover the variations of temperature with time during the production contribute to these imperfections. Previously and even to date the produced sound gears in many industries were tested by using the human ears to determine the defect gears and good gears, other methods like pitch detection method and faults detection method are applied. However those method have been found to be unreliable and therefore accelerated to bad sorting between good gears and bad gears. In this paper we proposed a powerful approach which used Digital signal processing techniques to determine the quality of the sound Gears. We integrated Correlation technique, Normalization and alignment methods. The techniques were applied to the extracted sound signals. The final extracted features (amplitudes, frequencies and phases) were classified and hence used to determine the good (non-defect) and bad (defect) gears. The entire process provided the perfect and higher accurate results and suggest that the tool can be used to identify good and bad sound gears.
\end{abstract}

Keywords: Signal processing techniques, sound Gears, signal features, Correlation, Computer Vision

\section{Introduction}

The advanced computer technology has facilitated the analysis, processing, decision making and production in various industries. However in some of computerized system the quality of the produced product may be low and therefore call for the need of higher intelligent tool for determination of the defect- product to separate from good ones. In this paper we introduced one of the powerful techniques for detection of the defect and non-defect sound product, Sound Gear has been our fundamental case study for development of the tool.

Gear is a toothed machine part, such as wheel with some groves at its surface coupled to either motor or engine to one part and connected to a toothed machine such as wheel or cylinder so as to transmit power or to change direction or speed of the motion [1], [2], [3].

Sound gear is the smallest and lightest electronic product on the market rests discreetly inside the wearer's ear to deliver natural wind reduction and superior sound quality [4].

The computer vision is the contemporary technology which involves transformation of data from a still or video camera into either a decision or a new representation for the purpose of achieving some particular features [5].

Digital signal Processing is an operation designed for extracting, enhancing, storing, and transmitting useful information [6]. DSP is the numerical manipulation of signals, involves measuring, filtering, reproducing or compressing of continuous analogy signal. It is characterized by representing signals as discrete time, discrete frequency, or other discrete domain signals. Different signal processing techniques such as Fourier transform, z-transform, Wavelet transform, convolution, and correlation to mention the few are used to realize the required process based on the information or features and the use of the information or features [4]

In this research paper, the sounds from sound gears were extracted, using anaudio technical-USB condenser Microphone, and stored to form a database. Digital signals processing techniques were employed to extract features and analyze the features, special threshold-classifier was used to features.

The decision making for the classifier was made based on Amplitude threshold set value. The approach based on the concept that the similarities and differences in soundsignal features (amplitudes, frequencies and phases) to determine the defect and non-defect gears

\section{Related Works}

There are numerous considerable methods which are used to ensure the produced products have higher quality to mention the few, several industries approach based on person ear in decision making for sound gears, it has been reported that many people came with different conclusions when they were assigned to determine the good ones and bad ones.

Also faults detection techniques are strongly used to identify products with faults and consider them as bad product Machine Vision Based Methods for Fault Detection

\section{1) Method 1: Fault Detection using GMMs and Blob Analysis}

This method uses the foreground detector system object to monitoring the machine while it is in operation and classifies the operating condition into a normal operation, a transfer 1 jam or transfer 2 jams. The foreground detector object reads grayscale video frames to compute and return the foreground mask using Gaussian mixture models (GMMs) (Stauffer, C. and Grimson, 2000). The single Gaussian model for background detection may not be reliable in case of lighting 


\section{International Journal of Science and Research (IJSR) \\ ISSN (Online): 2319-7064 \\ Index Copernicus Value (2013): 6.14 | Impact Factor (2014): 5.611}

changes and scene changes. Hence, the approach of adaptive Gaussian mixture model, where each pixel in the scene in modeled by a mixture of $\mathrm{K}$ Gaussian distributions works better for moving objects detection.[7] if $I$ is the image sequence and $\left\{x_{0,} y_{0}\right\}$ represent the location of a pixel then the history of the pixels value $x_{t}$ is obtained by

$$
\left\{X_{1}, \ldots \ldots, X_{t}\right\}=\left\{I\left(x_{0}, y_{0}, i\right): 1 \leq i \leq t\right\}
$$

The recent history of each pixel is modeled by a mixture of $\mathrm{K}$ Gaussian distribution. The probability of observing a certain pixel value $x_{t}$ at time $t$ can be written as

$$
P\left(X_{t}\right)=\sum_{i=1}^{K} \omega_{i, t} * \eta\left(X_{t}, \mu_{i, t}, \sum_{i, t}\right)
$$

Where $\omega_{i, t}$ the weight for ith Gaussian Component is, $\mu_{i, t}$ is the mean of the Gaussian component and $\eta\left(X_{t}, \mu_{s} \Sigma\right)$ is the normal distribution of $i^{\text {th }}$ component with $\mu$ as the mean and $\sum_{i}=\sigma_{i}^{2} I$ as the covariance

$$
\eta\left(X_{t}, \mu, \Sigma\right)=\frac{1}{2 \pi^{\frac{n}{2}}\left|\sum\right|^{\frac{1}{2}}} e^{-\frac{1}{2}\left(x-\mu_{t}\right)^{T}} \sum_{k}^{-1}\left(x-\mu_{k}\right)
$$

The updated weights are given by

$$
\begin{gathered}
\omega_{k, t}=(1-\alpha) \omega_{k, t-1}+\alpha\left(M_{k, t}\right) \\
\mu_{t}=(1-\rho) \mu_{t-1}+\rho\left(X_{t}\right) \\
\sigma_{t}^{2}=(1-\rho) \sigma_{t-1}^{2}+\rho\left(X_{t}-\mu_{t}\right)^{T}\left(X_{t}-\mu_{t}\right) \\
\rho=\alpha \eta\left(X_{t} \mid \mu_{k}, \sigma_{k}\right)
\end{gathered}
$$

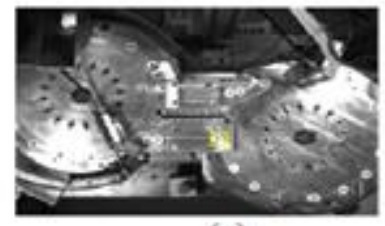

(a)

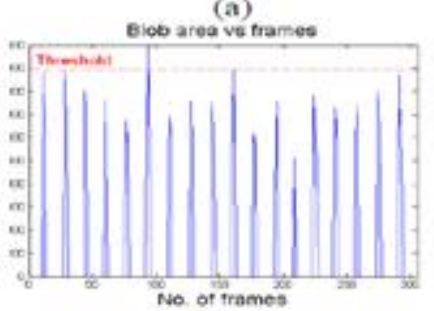

(d)

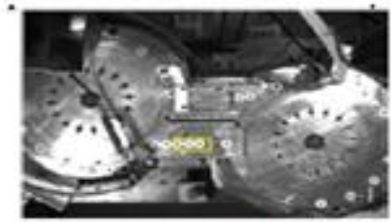

(b)

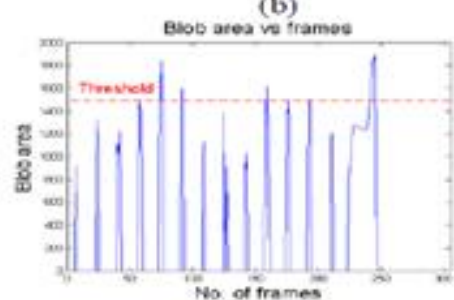

(e)

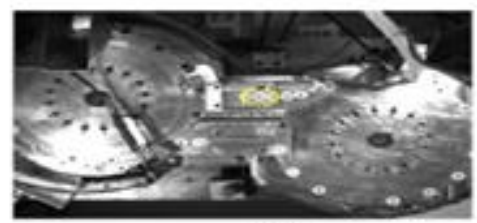

(c)

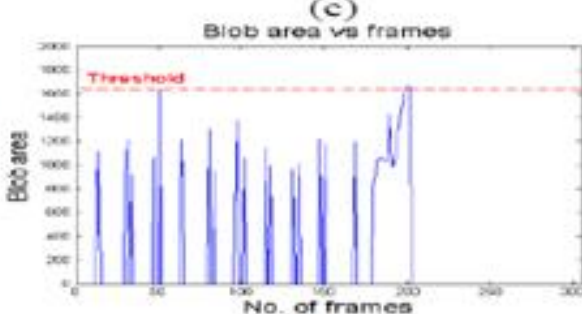

(f)

Figure 1: fauilts detections using blob and GMMs

2) Method 2: Fault Detection using Optical Flow Method Optical flow is a method popularly used to estimate motion from one frame of a video to another frame. It is a two dimensional vector given by equation (9) which is the measure of the motion in $\mathrm{x}$ and $\mathrm{y}$ directions respectively. [7]

$$
E=\iint\left(I_{x} u+I_{y} v+I_{t}\right)^{2} d x d y+\alpha \iint\left\{\left(\frac{\partial u}{\partial x}\right)^{2}+\left(\frac{\partial u}{\partial y}\right)^{2}+\left(\frac{\partial v}{\partial x}\right)^{2}+\left(\frac{\partial v}{\partial y}\right)^{2}\right\} d x d y
$$

However the computer vision based techniques for finding the faults are not reliable and applicable for the closed system and circuits hidden in some chips, because the techniques can work to all systems which their components can be seen by eyes but not hidden or intrinsic components which are highly affected by temperature and cause poor quality of the product

Digital Signal processing researchers were working for finding the similarities between the signals, under this concept it would be possible to find defect object especially for those sound object or system that gives signals and use the similarities to make decision the following were among the signal processing techniques used to find the similarities and differences between the sounds

\section{3) Spectral Models Related to the sinusoids and Noise Model}

Under this approach, they used additive synthesis as traditional sound synthesis method that is very close to the sinusoidal model. It has been used in electronic music for several decades [8]. It represented the original signal as a sum of sinusoids with time-varying amplitudes, frequencies and phase's .However, it did not make any difference between harmonic and non-harmonic components. Did not give any promising results to our need. Required very large amount of sinusoidal to represent non harmonic components

\section{4) Transient Modeling}

While sinusoids and noise were used to model a large variety of sounds, they performed poorly with very rapidly changing signals components and have a large bandwidth, the number of sinusoidal required is large. Also, the timeresolution used normally not well enough for transient. A transient detector determined where the transients are located and other parts of signal are represented with the parametric sinusoids and noise model, the detected transient is represented with non-parametric transforming coding. [9] .The transforming coding is strictly used for a short amount of time $(66 \mathrm{mS})$. Transient model has been used together with sinusoids and noise model in the system represented in [6]-[8]. It at end produced a sound coding scheme. However, that work gave no fundamental concept for construction of the system which can distinguish defect and non-defect gears 


\section{International Journal of Science and Research (IJSR) \\ ISSN (Online): 2319-7064 \\ Index Copernicus Value (2013): 6.14 | Impact Factor (2014): 5.611}

\section{5) Cross-Correlation method}

Sinusoids are defined as frequency components which have significantly more energy than the adjacent frequencies. Cross-Correlation (also called cross-covariance) between two input signals is a kind of template matching. [4] Crosscorrelation is done in any number of dimensions. The cross correlation uses the frequency components idea to calculating the cross correlation between the short-time spectrum of the signal and the spectrum resulting from the ideal sinusoid and sculling the result of the overall spectral shape. The obtained result is called the sinusoidal likeness measure. [6]- [11]-[12]-[13].The cross correlation method has been used successfully especially in speech coding [14], where the voicing index is similar to sinusoidal likeness measure

However cross correlation gives good information about how to phase one of the signals, for it to best match the other one, but it gave no explanation bout how similar they are. It has been observed that, cross correlation can be really affected by the signals amplitude, the higher amplitude in cross correlation dictates the higher similarity. Which was proven to be wrong in many cases including in cases of $\sin (x), 10 \sin (x)$. if $\sin (x)$ and $10 \sin (x)$ Are crosscorrelated, will yield large amplitude than, if sin $(x)$ is autocorrelated with $\sin (x)$, in this technique the one yielding large amplitude dictates the larger similarity and the other with small amplitude dictates small similarity, this concept gives wrong results, $\operatorname{since} \sin (x)$ and $\sin (x)$ are signals with $100 \%$ similarity, while $\sin (x)$ and $10 \sin (x)$ are not similar in amplitudes, therefore ,this scheme yet yield unpromising results [6],[11],[12],[13].[17]

\section{Proposed Approach}

The main purpose of this research is to establish the powerful and robust tool which detects the quality of the Sound Gear and classifies as the imperfect gear and perfect gear. A designated tool transforms the extracted sound signals from gears to the new presentable forms for processing and analyzing. From the analyzed features the designed tool automatically make decision on the class of the gear .fig 2 shows the block diagram for the sound signal processing. We have divided the developed algorithm into 4 stages.

\section{a) Pre-processing stage}

In this research, the pre-processing stage involves the sound Acquisition and storage. It is sometimes referred to the features extraction from signals, but in this case the sound from Gears are extracted / recorded by special recording Microphone named audio technical-USB condenser Microphone, The sample Gears used for extraction of sounds were produced in one of the company having an industry for earphones production, such gears were used as samples to facilitate the development of tool fig.1 (a) show the sample gear

To avoid the additive noises such as Radio frequency interferences, audio cables picking up Magnetic and Electrostatic hum fields radiated by power wiring in the walls of room, magnetic induction fields, the method proposed in [15] was employed of which the sounds of gears were recorded at the very silent closed box, with less radioactive interferences. The sounds were then stored in the form of sound waves in the computer with files of extension .wav

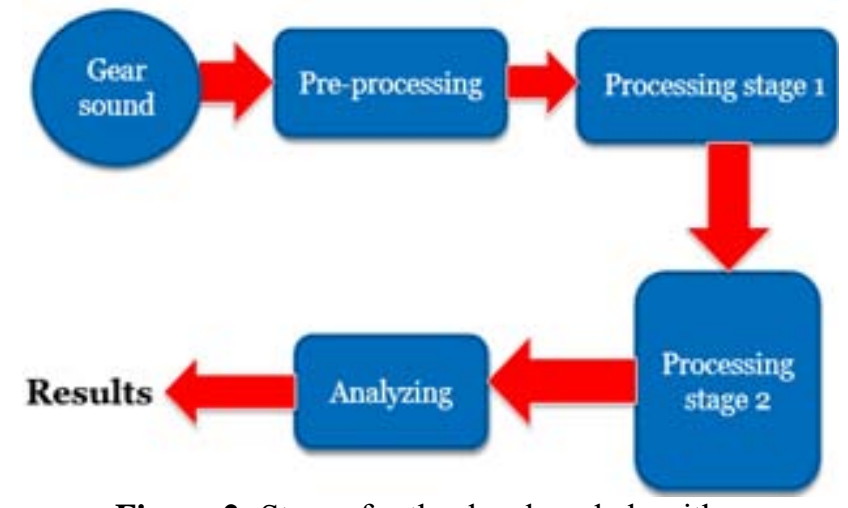

Figure 2: Stages for the developed algorithm

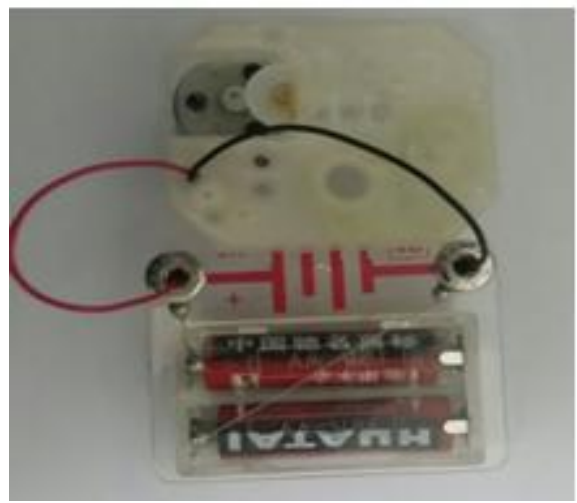

Figure 3: The top view of the Sound Gear used

\section{b) Processing stage 1}

The stage involves the presentation and processing techniques such Cross-correlation and auto-correlation.

\section{- Presentation}

The audio wave was transformed to the graphical presentation of the waves, which was then transformed to the sinusoidal signal presentation for better visualization [9], [14], [16]

- Cross-correlation

Once the waves have been presented to the sinusoidal forms, the cross -correlation technique was introduced. The characteristics such as amplitudes, frequencies and Phases were easily revealed [6], [11], [12], and [13]. This cross-correlation was between the referenced good sound signal and the unknown sound signal, the cross-correlation was defined by the following equation [6], [8].

$$
\begin{aligned}
& r_{x_{y} y}(l)=\sum_{n=-\infty}^{m} x(n) y(n-l) \\
& r_{x_{y} y}(l)=\sum_{n=1}^{N-[\mathbb{k} \mid-1} x(n) y(n-l)
\end{aligned}
$$

Index $l$ is called the shift or lag parameter.

\section{- Auto-correlation}

This was employed to the referenced signal itself for the purpose of understanding the signal behavior. It is the measure of the degree to which the signal is similar to itself .The following are the auto-correlation equations [6], [11].

$$
\begin{array}{r}
r_{x x}(l)=\sum_{n=-\infty}^{m} x(n) x(n-l) \\
r_{x}(l)=\sum_{n=1}^{\mathbb{N}-|\mathbb{k}|-1} x(n) x(n-l)
\end{array}
$$




\section{International Journal of Science and Research (IJSR) \\ ISSN (Online): 2319-7064}

Index Copernicus Value (2013): 6.14 | Impact Factor (2014): 5.611

The algorithm (9) was implemented in Mat lab because it support a finite duration.

\section{c) Processing stage 2}

It involves advanced signal processing techniques such as Normalization, and alignment.

\section{- Normalization}

Once the cross-correlated and auto-correlated results are obtained it is difficult to analyze their resembling,therefore Normalization technique is employed, there are frequency normalization and Amplitude normalization, we introduced the Amplitude normalization which set the amplitude of both Cross-correlation and auto-correlation to the unit value.

In additional to that frequency Normalization which was employed to facilitate the study of the signal characteristics in unit frequency. Both techniques introduced brought a clear view to the processed signals and enabled the understanding of the similarities and dissimilarities in all features. The normalization equation is simply given in equation (12) and (14) for auto-correlation and Crosscorrelation respectively

$$
\begin{aligned}
& \left|r_{x x}(0)\right| \leq r_{x x}(0)=E_{x} \\
& N\left(a_{s} f\right)=\frac{M\left(a_{0} f, j\right)}{M_{\max }(A, p b)} \\
& P_{x x}(0)=\frac{r_{x x}(d)}{r_{x x}(0)} \\
& \left|r_{x y}(1)\right| \leq \sqrt{r_{x x}(0) r_{y y}(0)}=\sqrt{E_{x} E_{y}} \\
& \rho_{x y}(l)=\frac{r_{x y}(D)}{\sqrt{r_{x x}(0) r_{y y}(0)}}
\end{aligned}
$$

- Alignment

It involved the synchronization of the results of the normalized cross-correlation and normalized autocorrelation to have the same starting point in time domain which plays a great role in studying of the, amplitude, phase, and frequency differences between the two results

\section{d) Enhancement and Analysis}

The aligned signal results are brought for visual observation to observe how close, the frequencies, phases and amplitudes between the normalized cross -Correlation and normalized auto-correlation are. In this research the differences in amplitudes of normalized cross -correlation and normalized auto-correlation were used to make the judgment for the similarities of the signals, and the results were interpreted to be the judgement for the quality of the Gear. It is expected the differences to be very small for all good gears. In this experiment the different is set be as small as less than 0.4. The formula for difference in amplitude applied is a follows.

$$
d_{\square}=A_{M N A r}-A_{M N C r}
$$

Where $A_{M N A Y}$ the maximum amplitude of the Normalized aligned Auto-correlated signal result, $A_{\text {MNCr }}$ is the Maximum amplitude of the Normalized aligned CrossCorrelated signal result, and $d_{\alpha}$ is the difference between the amplitude

\section{Experimental Results}

Based on analytical Digital processing algorithms employed to process the sounds to realize the imperfection and perfection of sound gears, from the initial stage to the final stage, the following are experimental sequential results, presenting the two sound gears of which one was defect gear sound processing procedures and the other was good gear sound processing Procedure

Summary for signal representations for both perfect and imperfect sound signals

Processing stages with imperfect sound with perfect sound

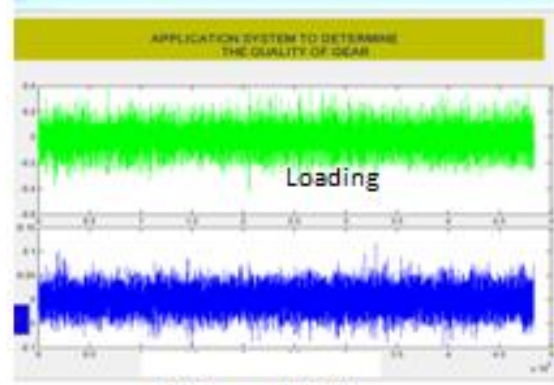

Figure (a) (i)

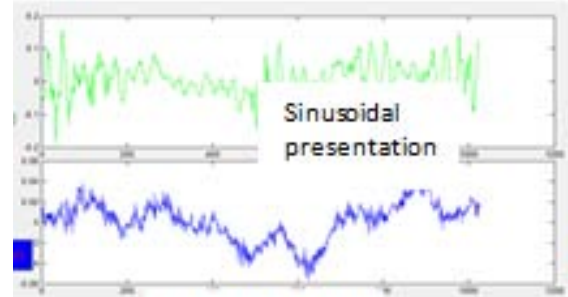

Figure (b) (i)

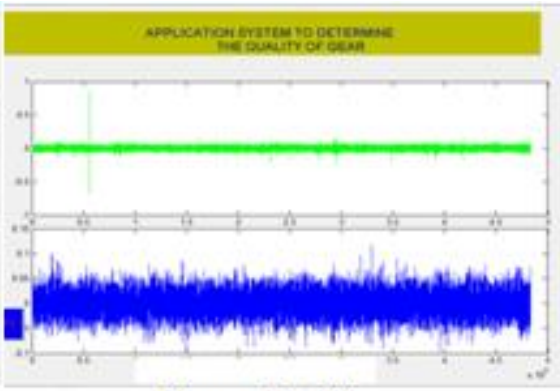

Figure (a) (ii)

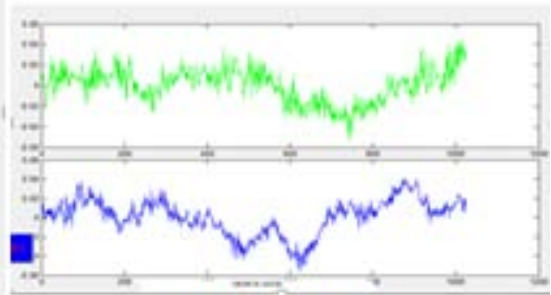

Figure (b) (ii) 
International Journal of Science and Research (IJSR)

ISSN (Online): 2319-7064

Index Copernicus Value (2013): 6.14 | Impact Factor (2014): 5.611

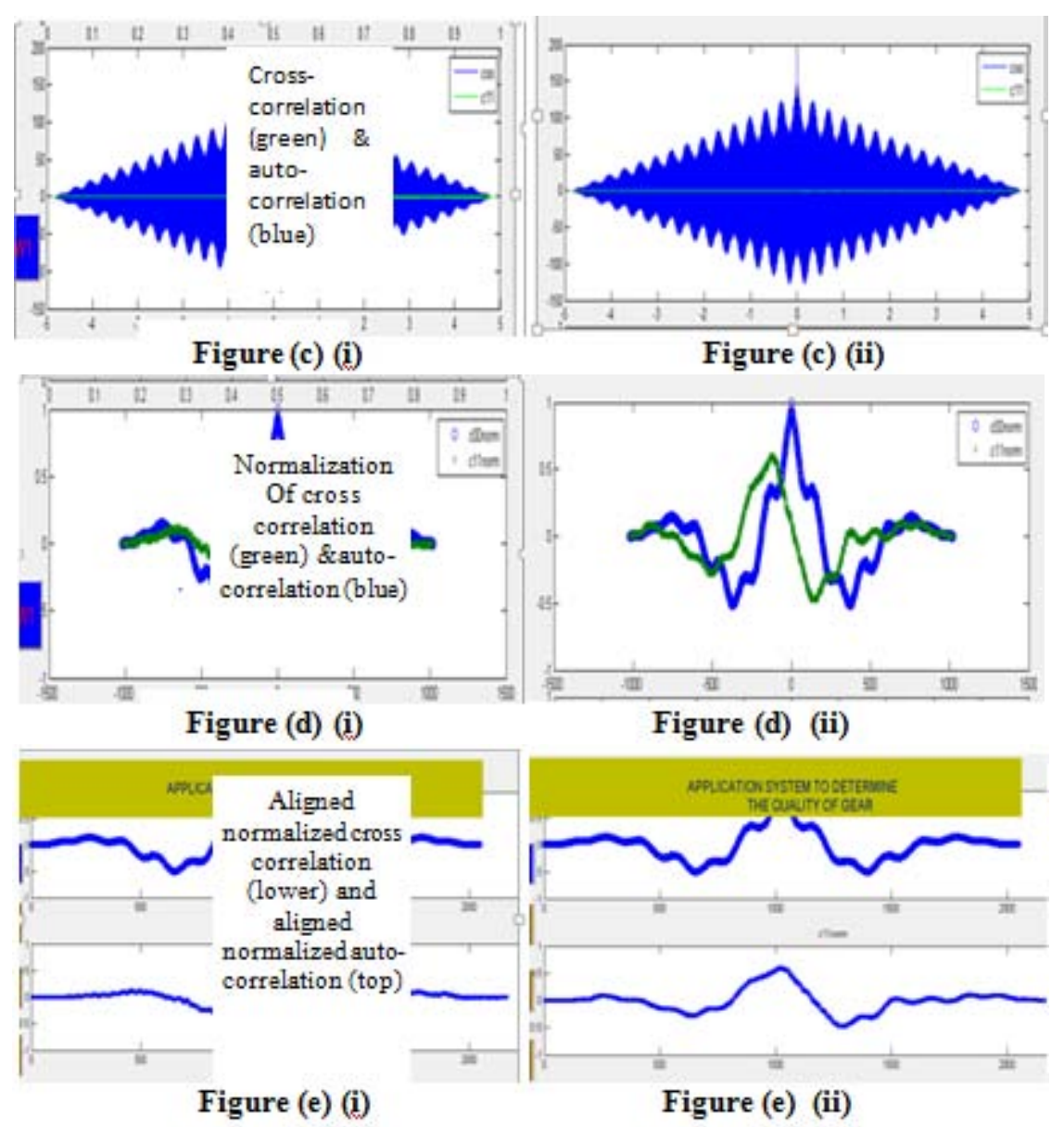

Table 1 for results of 10 test sound Gears

The last figure Fig (e)(i)reveals the mismatching between the aligned normalized cross-correlated and auto-correlated in terms of phase, and amplitude, the amplitudes, difference in amplitude dictated that sound signal was from defect Gear, i.e. $d_{a} \geq 0.4$

The same process from initial to the end was done for the good gears sound, after undergone through all stages, clearly the higher degree of similarity between the aligned normalized cross-correlated and Auto-correlated was observed in Fig $(e)(i i)$. In this case the $d_{a}<0.4$ which dictated that the sound was good and therefore the gear was perfect. In this research 8 imperfect Gears were provided, and 2 perfect gears and the results of the differences $d_{\alpha}$ were summarized in table 1

\section{Conclusion}

In this research we proposed the enhanced Digital signal processing techniques of which they are integrated in the computer mat lab Technology to develop a tool for the automatic detection of the Quality of the sound Gear .The approach had been found to have $100 \%$ accuracy after being tested for 10 sound gears in July 24, 2015 at the school electronic Engineering of which among the 8 gears were observed as bad gears and 2 gears classified as good gears. The choice of any of good gears to be the reference gear brought the same promising results for the detection, It is clearly revealed that the approach can suppress the problem of using human ear as the classifier for the good or bad gear, the developed tool guarantee the short time consuming and reliability.

Research involved the extraction of sound signals, presentation, cross-correlation, auto-correlation, Normalization, alignment as the basic flow techniques for development of the tool. However the finding of the amplitudes difference was the key to the classification.

The approach was efficient, real time and effective with low cost and less time consuming. The complete system developed as a tool found to be convenient and therefore can be installed in our general purpose computers for the classification of gears as to either defect or non-defect.

\section{Acknoledgement}

Benedictor Alexander sincerely conveys his remarkable thanks to his Father Mr. Alexander Longino for both financial and hearty support on matters concerning education and intellectual life. He also thanks Prof. Li li from school of Electronics Engineering of Tianjin University of Technology and Education for ideal support toward accomplishment of this paper.

\section{References}

[1] engk12.ece.missouri.edu/documents/matl/S2IGearsPresentation.ppt, Robotics Academy 2002. 


\section{International Journal of Science and Research (IJSR) \\ ISSN (Online): 2319-7064}

Index Copernicus Value (2013): 6.14 | Impact Factor (2014): 5.611

[2] Engineering Information Boston Gear, www.bostongear.com/pdf/gear_theory.pdf

[3] Fundamentals of Machine Elements, . $3^{\text {rd }}$ ed. Schmid, Hamrock and Jacobson_ C 2014 CRC Press

[4] SoundGear-Professional-Brochure.pdf

[5] L. Robert, Opencv2computer Vision Application Programming Cookbook, Packt Publishing Ltd 32, Lincoln Road Olton Birmingham, B27 6PA, UK, 2011.

[6] Vinay K.Ingle, John G.Proakis "Digital Signal Processing, by using MATLAB (Third Edition)", 2012(book style)

[7] "A Comparative Study of Machine Vision Based Methods for Fault Detection in an Automated Assembly Machine" 43rd Proceedings of the North American Manufacturing Research Institution of SMEhttp://www.sme.org/namrc,VolumeXXX, 2015,Pages $1-13$

[8] Ali, M. "Adaptive Signal Representation with Application in Audio Coding". Ph.D. thesis, University of Minnesota,1996 (Thesis style)

[9] Levine, Scott "Audio Representation for data Compression and Compressed Domain Processing "Ph.D. Thesis .Stanford University, 1998 .(Thesis style)

[10] Brown, Judith C."Calculation of a constant Q Spectral transform". Journal of Acoustic Society of America, Vol 89(1), January 1991.(paper style)

[11] John G. Proatis, Dimitris G. Manolakis , Digital Signal Processing, Principles, Algorithms, and Applications ,Third Edition, 1996 (book style )

[12] Douglas Lyon, "The Discrete Fourier Transform, Part 6: Cross-Correlation", Journal of object technology, Published by ETH Zurich, Chair of Software Engineering (CJOT, 2010 ,Vol. 9, No. 2, March - April 2010(Paper style)

[13] Ammar Abu-Hudrouss, Islamic University Gaza, Crosscorrelation and Auto-correlation spring $2008 \mathrm{ppt}$

[14] Griffin, D. \&Lim, J."A New Model-Based Speech analysis /Synthesis System ".IEEE International Conference on Acoustic, Speech and Signal Processing Tempa, Florida 1985. (Conference style

[15] http://www.prosoundweb.com/article/preventing_hum and_rfi_in_your_studio (preventing HUM and RFI)

[16] Bergman, Albert. S."Auditory Scene Analysis" .MIT P29Press, 1990. (Conference style)

[17]E1.10 Fourier series and Transforms (20155585), Transform - Correlation: $8-1 / 11$ (ppt)

[18] 18Bu-ChinWang, Digital Signal Processing Techniques and Applications in Radar Image Processing, August 2008

[19] Colomes, C. \& Lever, M. \& Rault J.B. \& Dehery Y.F. "A Perceptual., Colomes et al. 1995(book style)

[20] Brown, Judith C. \& Puckett, And Miller S. "An efficient algorithm for the calculation of a constant Q transform". Journal of Acoustic Society of America, Vol 92(5), November 1992. (Paper style)

\section{Author Profile}

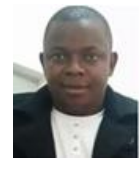

Benedictor Alexander was born in Lindi Region ofTanzania, East Africa in June, 1989. He attained Bachelor's Degree of Science in Electronics and Communication from University of Dares salaam, Tanzania in 2013. He then worked as IT and Technical supporter for almost 1 year before joined Tianjin University of Technology and Education in China for his further studies. He is recently pursuing master's degree of science in signal and information processing engineering at Tianjin University of Technology and Education. His research interests include development of mathematical models, Digital signal processing, biomedical, pattern recognition, artificial neural networks and intelligent systems, based on image processing and Biomedical engineering. $\mathrm{He}$ is famous for for one of his published paper SUB158660, Volume 10, Issue 4, October, 2015 "Fastest iteration Method for Estimation of the solution for non-linear function $f(\mathrm{x})=$ 0 (Bennie's Method)" 